\title{
Aprendiendo a enseñar Didáctica de las Matemáticas en el Grado de Educación Primaria
}

\author{
ANA ESCUDERO-DOMÍNGUEZ \\ Universidad de Sevilla \\ Facultad de Ciencias de la Educación \\ Departamento de Didáctica de las Matemáticas \\ aescudero1@us.es \\ ORCID: https://orcid.org/0000-0002-1620-3852 \\ D.O.I.: http://dx.doi.org/10.12795/JDU.2018.i01.12 \\ Pp.: 216-229
}

\section{Resumen}

Se presenta una experiencia llevada a cabo en la asignatura de Didáctica de las Matemáticas del Grado de Educación Primaria. No es la primera vez que se intenta llevar a cabo un ciclo de mejora en esta asignatura, sino que ya se han realizado otros ensayos anteriores en ésta. Dicho grado debe proporcionar a los estudiantes una formación universitaria orientada a favorecer el papel activo y emprendedor del alumno (Escudero-Domínguez, 2017). El objetivo de este ciclo de mejora, al igual que en los otros, es convertir el proceso de enseñanza-aprendizaje en algo dinámico y participativo. En el desarrollo de este trabajo se siguió una metodología basada en el empleo de problemas y casos prácticos, para alcanzar un aprendizaje más significativo. Los resultados muestran valoraciones positivas que se reflejan a modo de escaleras de aprendizaje. 
Palabras clave: Ciclo de mejora, Didáctica de las Matemáticas, Docencia Universitaria, Grado de Educación Primaria, Experimentación Docente Universitaria.

\section{Contexto de la intervención}

Se presenta un ciclo de mejora desarrollado en el Grado de Educación Primaria, concretamente en la asignatura de Didáctica de las Matemáticas. Este ciclo de mejora es la continuación de otras experiencias de innovación que hemos realizando durante varios cursos anteriores. En esta asignatura es la tercera vez que voy a aplicar un ciclo de mejora. A continuación, comento a grandes rasgos lo que he tratado en los ciclos anteriores. En el primero abarqué uno de los módulos que he vuelto a tratar en éste, geometría. Y el segundo ciclo realizado incorporé varios módulos del primer cuatrimestre que versan sobre el número.

La asignatura de Didáctica de las Matemáticas es una asignatura obligatoria de 2o curso del Grado de Educación Primaria donde encontramos un alumnado que ya ha cursado otra asignatura obligatoria sobre matemáticas, Matemáticas Específicas para Maestros. Esta asignatura está dividida en distintos bloques de contenidos, de los que voy a realizar mi ciclo de mejora atendiendo a los últimos: geometría y probabilidad y estadística.

Sobre el módulo de geometría ya he realizado anteriormente un ciclo de mejora. Vuelvo a insistir sobre él ya que considero que esta materia suele ser vista como un proceso memorístico, sin comprender lo que se pretende enseñar, lo que les genera inseguridad. Esto hace que le suelan dedicar poco tiempo e incluso lleguen a ignorarla (Escudero-Domínguez y Carrillo, 2014).

Al otro módulo elegido, Probabilidad y Estadística, le ocurre un poco lo mismo, no suele alcanzar en las aulas la misma importancia que, por ejemplo, el bloque de números. Con lo que nos planteamos afianzar los conocimientos de los futuros docentes para que estos adquieran los 
conocimientos deseados sobre esta parte de las matemáticas, y no sientan miedo o limitación a la hora de impartirlos en el aula.

\section{Diseño del ciclo de mejora docente}

\section{Modelo metodológico}

En el desarrollo del ciclo de mejora docente se siguió una metodología de investigación-acción, intentando evitar la exposición de lecciones magistrales, proponiendo que sean los alumnos los que construyan el conocimiento, cediéndoles la responsabilidad (Carrillo, Climent, Gorgorió, Prat y Rojas, 2008). El modelo metodológico que pretendo seguir es el siguiente:

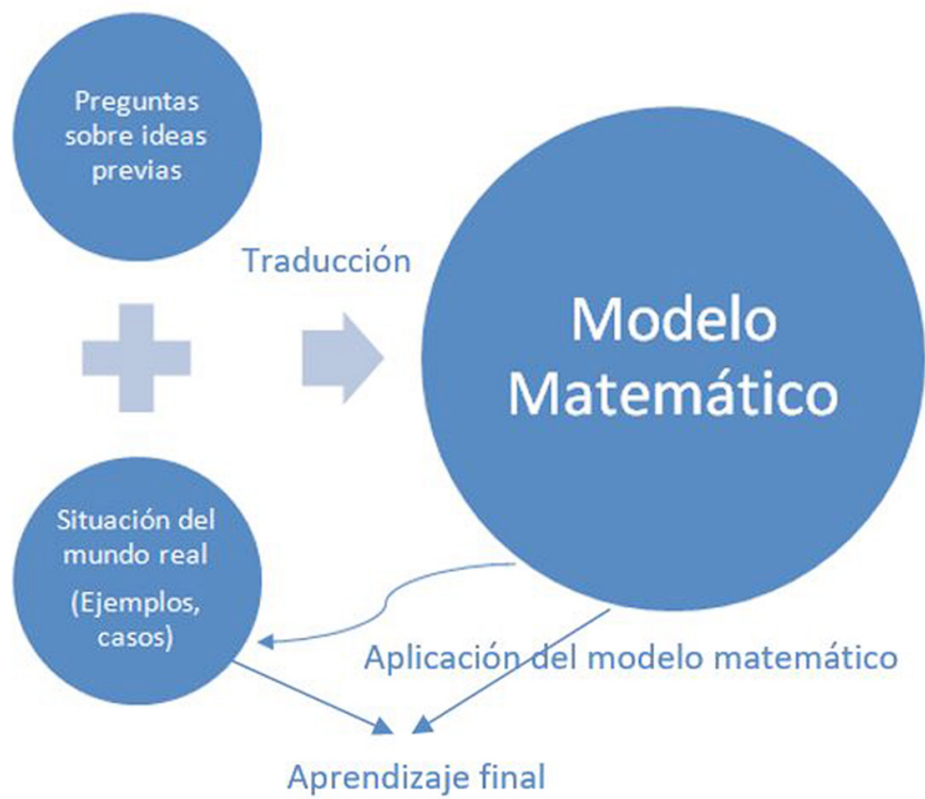

Figura 1. Modelo metodológico llevado a cabo

La forma de trabajo que propongo es comenzar por realizar preguntas sobre las ideas previas del alumnado

Jornadas de Formación e Innovación Docente del Profesorado I № 1 (2018)
Esta obra se distribuye con la licencia Creative Commons 
referente a contenidos de cada bloque para poder conocer qué saben sobre éste, y ver de dónde puedo partir. A continuación, se les presentan distintas situaciones del mundo real, apoyándonos en distintas lecturas, en material audiovisual o mediante transcripciones de casos reales. Una vez hayan interiorizado lo anterior, les pediremos que intenten hacer una abstracción de ello, es decir, pasar a lo que sería el modelo matemático (teórico) ayudándonos de nuevo de los ejemplos tratados anteriormente como aplicación del modelo matemático. Este último proceso nos conduce al aprendizaje final favoreciendo en los estudiantes la capacidad para aprender por sí mismos.

Para finalizar, vamos a mostrar la idea de modelo metodológico ideal que tengo, donde intento alcanzar, tanto un modelo basado en la reelaboración de las ideas de los estudiantes (Figura 2), como el modelo consistente en formular un problema de la vida cotidiana o situación técnica, resolverlo si es posible e interpretar los resultados en términos del problema y de la situación planteada, apoyado en la modelización matemática (Gómez, 2006) (Figura 3).

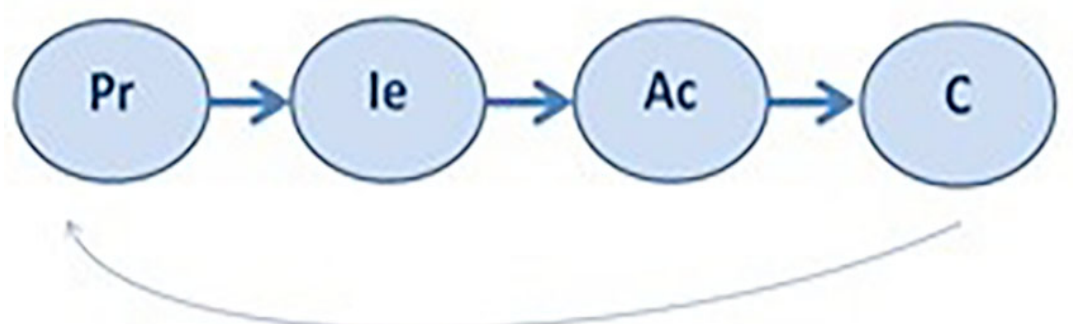

Figura 2. Esquema de modelo metodológico basado en la reelaboración de las ideas de los estudiantes. Tomado de De Alba y Porlán (2017, p. 41)

Jornadas de Formación e Innovación Docente del Profesorado | № 1 (2018) Esta obra se distribuye con la licencia Creative Commons 
(4) Comparación

ESQUEMA DE PROCESO DE MODELIZACION

SITUACION DEL MUNDO REAL

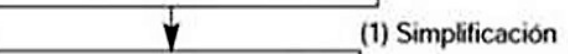

MODELO DEL MUNDO REAL

(2) Traduccion

MODELO MATEMATICO

(3) Aplicación de métodos matemáticos

CONCLUSIONES

Figura 3. Esquema del proceso de modelización.

Tomado de Gómez (2006, p. 72).

\section{Mapa de contenidos y problemas clave}

Los contenidos que quiero abordar en este ciclo de mejora están centrados, por un lado, en geometría y, por otro, en probabilidad y estadística. Dado el espacio reducido de esta comunicación muestro solo una parte de éstos, basados principalmente en estadística.

Nuestro mapa de contenidos tiene como clave una serie de cuestiones como las que siguen: ¿Qué enseñar?, ¿Cómo aprende el alumnado? y ¿Cómo enseñar?

Al intentar dar respuesta a estas preguntas siempre me surge la misma inquietud, ¿dará tiempo? Muchas investigaciones como por ejemplo la de Grueso, Prado-Gotor y Pérez-Tejada (2014), nos insisten en que todos los contenidos no pueden enseñarse, sino que el maestro debe reflexionar cuáles son los contenidos organizadores que dan sentido y conforman al resto.

En el departamento solemos dividir cada bloque de contenidos en tres grandes apartados, por tanto, tendremos siempre estas tres partes diferenciadas en nuestro esquema, que son: contenido, aprendizaje y enseñanza. A continuación, mostraré el esquema de contenidos y la planificación de las sesiones del ciclo de mejora de estadística (figura 4). 

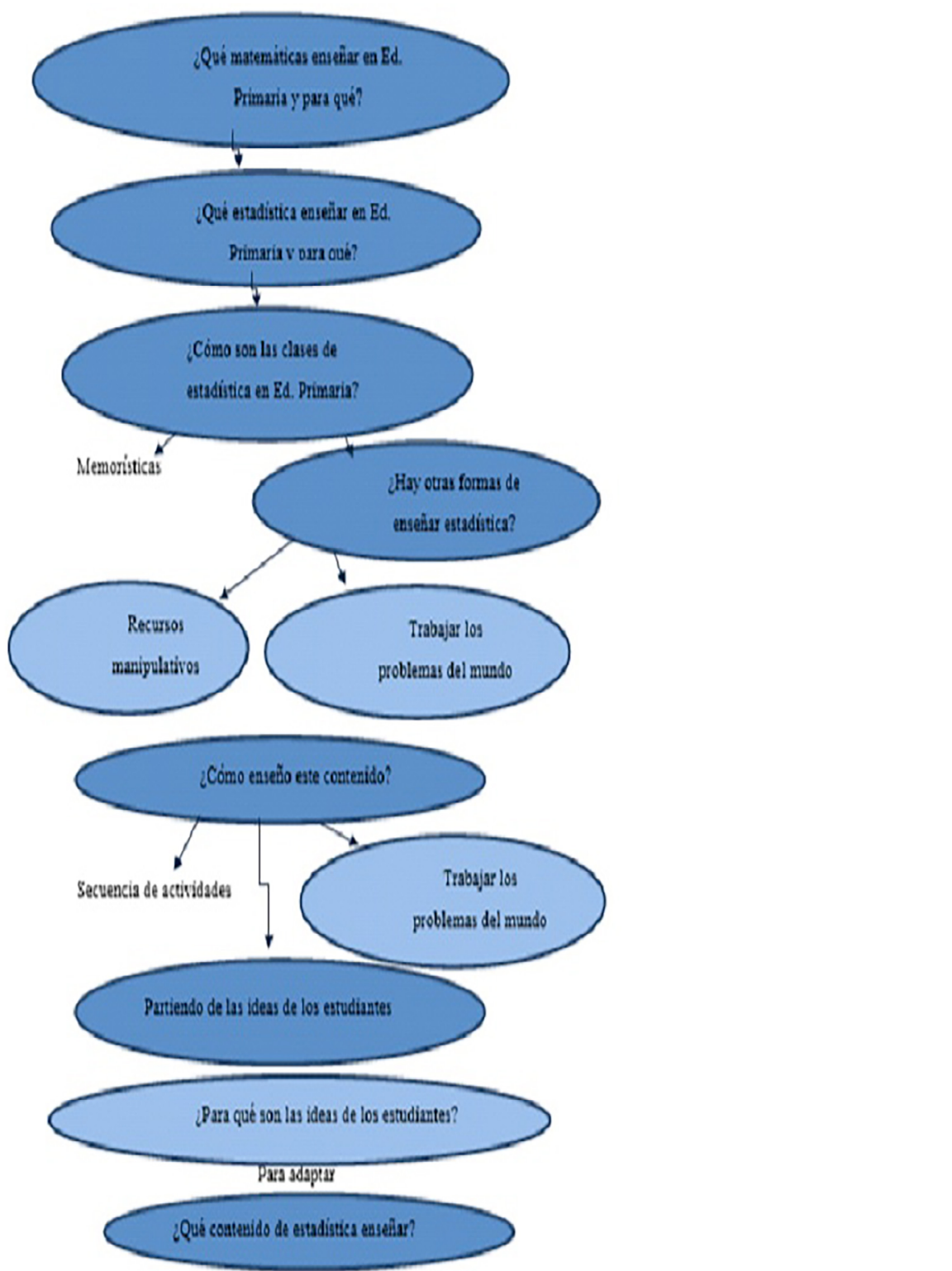

Conocimiento itil para !a wida

QQué seleccion de coatenidos lasen?

Figura 4. Mapa general de contenidos estadísticos en Ed. Primaria (basado en mapa general de contenidos y problemas de Navarro, 2017, p. 148)

Jornadas de Formación e Innovación Docente del Profesorado | № 1 (2018)

(c) (7) $\odot$ Esta obra se distribuye con la licencia Creative Commons 
¿Qué enseñar sobre estadistica?

- Estadística

- Tipos de estadística (descriptiva e in ferencial)

-Datos, gráficos

- Variación

- Distribución

- Muestreo
¿Cómo aprende el

alumnado la estadistica?

- Errores y dificultades en la comprensión de los conceptos estadísticos fundamentales

- Niveles de comprensión de los gráficos
¿Cómo enseñar la estadistica?

- Construir e interpretar gráficos

-Materiales y recursos

- Construcciones de situaciones de enseñanza/aprendizaje

Figura 5. Esquema de contenidos de estadística

\section{Secuencia de actividades}

Siguiendo el modelo metodológico y los mapas de contenidos descritos, a continuación, se expone el cronograma desarrollado en este ciclo de mejora elaborado para el bloque de estadística.

\section{Tabla 1}

Cronograma desarrollado para el bloque de estadística

\begin{tabular}{|l|}
\hline \multicolumn{1}{|c|}{ Descripción de las sesiones dedicadas a estadística } \\
\hline $\begin{array}{l}\text { S1. Presentación y recogida de información sobre las ideas previas que po- } \\
\text { seen los alumnos sobre estadística en Ed. Primaria (Anexo 1. Cuestionario). } \\
\text { El cuestionario dispone de una serie de preguntas que son las mismas que } \\
\text { se van trabajando en el desarrollo de las clases. }\end{array}$ \\
\hline $\begin{array}{l}\text { Lluvia de ideas sobre el significado de estadística. Entre todos, buscan } \\
\text { ejemplos y recordamos la definición. }\end{array}$ \\
\hline Recordamos vocabulario usual de esta parte de la materia. \\
\hline $\begin{array}{l}\text { Profundización acerca de la estadística, realizando un esquema con todas } \\
\text { las nociones requeridas sobre ésta para Educación Primaria. Al inicio como } \\
\text { una lluvia de ideas y despues, apoyados en la lectura de Batanero (2013), } \\
\text { para lograr un listado junto a su definición más exhaustiva. }\end{array}$ \\
\hline $\begin{array}{l}\text { S2. Mostramos distintos vídeos sobre estadística con situaciones del } \\
\text { mundo real. A continuación, realizamos un debate en torno a pregun- } \\
\text { tas como las que siguen: De lo anterior, ¿Qué crees que se debe ense- } \\
\text { ñar en Ed. Primaria? ¿Cómo lo enseñarías? ¿Hay otras formas de enseñar } \\
\text { estadística? }\end{array}$ \\
\hline
\end{tabular}

Jornadas de Formación e Innovación Docente del Profesorado | № 1 (2018) Esta obra se distribuye con la licencia Creative Commons Reconocimiento-NoComercial-SinObraDerivada Internacional (CC BY-NC-ND 4.0.) 
Intentamos hacerles entender que en la estadística existen dos ramas, descriptiva e inferencial, pero que en Ed. Primaria solo nos quedamos con la descriptiva

Sondeo mediante las siguientes preguntas: ¿Cómo aprende el alumnado la estadística? ¿Podemos distinguir fases en el aprendizaje?

En gran grupo intentamos hacer un listado de dificultades y/o errores en el aprendizaje de la estadística

S3. Intentamos establecer unas orientaciones sobre cómo ayudar a los niños en el desarrollo del razonamiento estadístico, ¿Cómo enseño este contenido?

Indagamos sobre los distintos materiales y recursos para trabajar la estadística en la escuela. Ellos buscan en sus ordenadores o móviles los posibles materiales y, finalmente, llegamos a un consenso.

Presentación de diferentes vídeos o situaciones que van mostrando niveles de comprensión de gráficos según la edad del alumnado.

S4. Diseñamos, entre todos, algunas situaciones de enseñanza/aprendizaje de la estadística. Lanzamos preguntas como: ¿Qué contenido de estadística enseñar?, ¿Qué selección de contenidos hacer?

Presentación y recogida de información de los alumnos sobre lo que ahora conocen sobre estadística (Anexo 1. Cuestionario)

\section{Aplicación del ciclo de mejora docente}

\section{Relato de una de las sesiones dedicadas a estadística}

Como he comentado anteriormente, me voy a centrar en la última parte del temario de la asignatura, concretamente en el bloque de estadística. Describo aquí la primera sesión dedicada a ésta. Comienzo la clase lanzando la siguiente pregunta: ¿Qué estadística enseñar en Ed. Primaria y para qué? A continuación, paso el cuestionario inicial, que recoge preguntas como la anterior, que se van a ir trabajando a lo largo del desarrollo del tema. Con este cuestionario espero obtener las ideas previas que poseen los alumnos de forma individual, ya que pienso que así 
puedo obtener mayor información que si lo hiciera en voz alta, además de la ventaja de tenerlo recogido, para poder observar qué han aprendido tras la instrucción realizada.

Tras recoger el cuestionario, les pregunto acerca de la definición de estadística. Realizamos una lluvia de ideas sobre el significado de estadística. Los alumnos van construyendo la definición y ofreciendo ejemplos de ésta. Con esta actividad van refrescando vocabulario usual de esta parte de las matemáticas. A continuación, les pido que, a modo de debate, reflexionen acerca de las nociones de estadística que se deben enseñar en Ed. Primaria y para qué. Finalmente, nos apoyamos en el currículo y en la lectura de Batanero (2013), para hacer un listado más exhaustivo.

Durante la sesión debo reconocer que los alumnos mostraron una actitud positiva, interesándose y prestando atención a las distintas actividades planteadas. La participación fue numerosa, aunque un grupo de alumnos monopolizaron las respuestas y otro se dedicaba a prestar atención y/o asentir con la cabeza. Los estudiantes planteaban sus ideas y se iban rebatiendo, algunas veces entre ellos y muchas otras era yo las que las refutaba. He observado que impartiendo este tipo de clase, aumento el interés de los estudiantes por la materia y logro conectar casi con la mayoría de éstos.

Evaluación del aprendizaje de los estudiantes

Para la evaluación del aprendizaje de los estudiantes utilicé un cuestionario de preguntas abiertas (Anexo 1) que pasé al inicio y volví a pasar una vez finalizada la docencia de esa parte de la asignatura.

Una vez recogida la información del cuestionario inicial procedí a analizarla, observando de forma pormenorizada las ideas previas que tenían los alumnos, así como las dificultades que mostraban éstos. Estas ideas previas se correspondían con las que me había encontrado en clase, en las que solo un $10 \%$ del alumnado se acercaba a la respuesta correcta. 
Al finalizar la materia pasé de nuevo el mismo cuestionario y comparé los resultados obtenidos. En este sentido, la evaluación fue positiva ya que se obtuvieron mejores puntuaciones, ahora sobre el $70 \%$ de la clase se acercaba a la respuesta correcta.

Para poder analizar las respuestas de los estudiantes, establecí 3 niveles de respuesta en cada pregunta, siendo el más completo el que había definido como correcto, es decir, el que mostraba todos los contenidos que me había propuesto enseñar (Navarro, 2017). Paso a mostrar una escalera de aprendizaje (Tabla 2).

\section{Tabla 2}

Ejemplo de escalera de aprendizaje en el módulo de Estadística

\begin{tabular}{|l|l|l|}
\hline \multicolumn{3}{|c|}{ ¿Qué nociones de estadística enseñar en Ed. Primaria? } \\
\hline Cuestionario inicial & \multicolumn{1}{|c|}{ Respuestas } & Cuestionario final \\
\hline $\begin{array}{l}\text { Nivel 3 } \\
5 \%\end{array}$ & $\begin{array}{l}\text { Resumir conjuntos de datos } \\
\text { mediante tablas, saber inter- } \\
\text { pretar los gráficos y extrapolar- } \\
\text { los a la realidad }\end{array}$ & $\begin{array}{l}\text { Nivel 3 } \\
70 \%\end{array}$ \\
\hline $\begin{array}{l}\text { Nivel 2 } \\
15 \%\end{array}$ & $\begin{array}{l}\text { Enseñar contenidos que nos } \\
\text { permitan resolver problemas de } \\
\text { la vida cotidiana }\end{array}$ & $\begin{array}{l}\text { Nivel 2 } \\
20 \%\end{array}$ \\
\hline $\begin{array}{l}\text { Nivel 1 } \\
80 \%\end{array}$ & $\begin{array}{l}\text { Contenidos mínimos marcados } \\
\text { por la legislación }\end{array}$ & $\begin{array}{l}\text { Nivel 1 } \\
10 \%\end{array}$ \\
\hline
\end{tabular}

\section{Evaluación del ciclo de mejora docente llevado a cabo}

Aunque normalmente la evaluación se concibe como un esquema que nos permite conocer la evolución del conocimiento de los estudiantes, no podemos olvidar que siempre debemos evaluar nuestra puesta en práctica (Escudero-Domínguez, 2017). Para ello elaboré otro cuestionario para conocer las opiniones de los estudiantes sobre el 
proceso de enseñanza y aprendizaje. Este cuestionario era de tipo escala Likert excepto la última pregunta que era de respuesta abierta, donde les preguntaba qué elementos creían que se podrían mejorar del proceso de enseñanza/ aprendizaje de esta asignatura. De éste obtuve un grato mensaje, del que puedo decir que casi el $90 \%$ de los estudiantes se encontraba entre satisfecho y muy satisfecho con la impartición de la asignatura.

También he de decir que para mí ha sido una experiencia muy gratificante, ya que he logrado conectar con casi todos los estudiantes y creo que todos ellos han aprendido. Para el próximo curso me gustaría mantener este tipo de clases, en las que prima el debate y a partir de éste intento ampliar los conocimientos de los estudiantes, conectando a su vez con las ideas previas que conocen sobre estos. También me gustaría introducir cambios en mi próximo ciclo de mejora como el no perder mucho tiempo sobre las preguntas de inicio pues las respuestas las tengo también en el cuestionario inicial, el intentar ajustar mejor los tiempos en las secuencias de actividades e incluso arriesgarme a realizar alguna clase invertida.

\section{Conclusiones}

El ciclo de mejora realizado ha supuesto una interesante experiencia ya que nuevamente me ha demostrado que otra forma de impartir clase es posible. Ciertamente me supone un reto organizar debates, ya que no sé cómo saldrán y si llegaré a obtenerle todo el partido que debería, pero es algo de lo que cada día estoy más concienciada en que son necesarios. Estos, además de captar la atención de todo el alumnado, son un buen conductor para fortalecer o ampliar los conocimientos de los estudiantes. Al hilo de lo anterior, debo resaltar la carga de trabajo del profesorado anterior a la puesta en práctica del debate, ya que debes de tener una serie de vías previstas 
para poder ir encaminando al alumnado hacia los contenidos que se pretenden enseñar.

Por otro lado me gustaría destacar, tras la experiencia de estos ciclos de mejora realizados, algunos de los principios didácticos que marcan mi proceso de aprendizaje/ enseñanza:

- Previo a cada módulo diseño un cuestionario que paso en la primera sesión. Este me permite ir adaptando las explicaciones a los conocimientos de los que parte el alumnado.

- Organizo las sesiones y secuencia de actividades de manera previa al desarrollo del módulo, aunque siempre se pueda reformular una vez comenzada su instrucción.

- Propongo tareas que abarquen los tres tipos de contenidos (conceptuales, procedimentales y actitudinales), y aunque inconscientemente le concedo más peso a los dos primeros, nunca me olvido de inculcar el respeto, fomentar el diálogo y la cooperación.

- Intento organizar secuencias de actividades que acerquen al alumnado a su futura profesión, aunque pienso que puedo mejorar esta secuencia introduciendo actividades investigativas y/o organizando el contenido por problemas prácticos para los alumnos, que les permita ir resolviendo cuestiones.

- Pretendo fomentar el interés del alumnado, dándole más tiempo a éste para que trabaje y se cuestione los contenidos, potenciando así su razonamiento crítico.

- Debo intentar proponer distintas actividades en torno a contenidos que suelan quedar menos claros, de forma que los alumnos puedan superar los obstáculos que puedan poseer.

- Con respecto a la evaluación del alumnado, mi propuesta es tener en cuenta la participación y las tareas desarrolladas en clase, además del examen de la asignatura. 
- Al finalizar la asignatura proponer un instrumento para mi evaluación, ya que esto me sirve bastante para tenerlo en cuenta en un futuro.

- Incluir una autoevaluación del alumnado con respecto a los casos prácticos.

\section{Referencias bibliográficas}

Batanero, C. (2013). Sentido estadístico: Componentes y desarrollo. I Jornadas Virtuales de Didáctica de la Estadística, la Probabilidad y la Combinatoria. Granada.

Carrillo, J., Climent, N., Gorgorió, N., Prat, M. y Rojas, F. (2008). Análisis de secuencias de aprendizaje matemático desde la perspectiva de la gestión de la participación, Enseñanza de las Ciencias, 26(1), 67-76.

De Alba, N. y Porlán, R. (2017). La metodología de enseñanza. En R. Porlán (Coord.), Enseñanza Universitaria. Cómo mejorarla (pp. 37-54). Madrid: Editorial Morata.

Escudero-Domínguez, A. (2017). Ciclo de mejora en Didáctica de las Matemáticas. IV Jornadas de Formación e Innovación docente de la Universidad de Sevilla (pp. 182-193). Sevilla.

Escudero-Domínguez, A. y Carrillo, J. (2014). Conocimiento matemático sobre cuadriláteros en estudiantes para maestro. En M. T. González, M. Codes, D. Arnau y T. Ortega (Eds.), Investigación en Educación Matemática XVIII (pp. 267-276). Salamanca: SEIEM.

Gómez, J. (2006). De la tradición a la innovación docente en matemáticas. Revista Técnica Industrial, 256, 7075<http://www.tecnicaind ustrial.es/TIAdmin / Nume$\mathrm{ros} / 16 / 43 /$ a43.pdf $>$ [consultado el 10 de mayo de 2018]

Grueso, E. M., Prado-Gotor, R. y Pérez-Tejada, P. (2014). Aplicación de un modelo didactico alternativo a la asignatura de fisicoquímica. I Seminario Iberoamericano de Innovación Docente de la Universidad Pablo de Olavide. Sevilla.

Navarro, E. (2017). Un ciclo de mejora en la formación inicial de profesores de Ciencias Sociales de Secundaria. En R. Porlán (Coord.), Enseñanza Universitaria. Cómo mejorarla (pp. 145-158). Madrid: Editorial Morata. 


\section{Cuestionario inicial-final para hacer un seguimiento de la evolución de los estudiantes en relación con los problemas clave}

\begin{tabular}{|l|}
\hline ¿Qué matemáticas enseñar en Ed. Primaria y para qué? \\
\hline ¿Qué entendemos por estadística? \\
\hline ¿Qué nociones de estadística enseñar en Ed. Primaria y para qué? \\
\hline ¿Cómo aprende el alumnado de Ed. Primaria la estadística? \\
\hline ¿Podríamos distinguir fases en su aprendizaje? \\
\hline $\begin{array}{l}\text { Realizar un listado de dificultades o errores que creéis que pueden tener } \\
\text { los estudiantes en el aprendizaje de la estadística }\end{array}$ \\
\hline ¿Cómo enseñar la estadística? \\
\hline $\begin{array}{l}\text { Establecer una serie de orientaciones sobre cómo ayudar a los niños en el } \\
\text { desarrollo del pensamiento estadístico }\end{array}$ \\
\hline $\begin{array}{l}\text { Nombrar y describir algunos materiales y/o recursos para trabajar la esta- } \\
\text { dística en Ed. Primaria }\end{array}$ \\
\hline Diseña alguna situación de enseñanza/aprendizaje de la estadística \\
\hline
\end{tabular}

Jornadas de Formación e Innovación Docente del Profesorado | № 1 (2018) Esta obra se distribuye con la licencia Creative Commons Reconocimiento-NoComercial-SinObraDerivada $\quad 4.0$ Internacional (CC BY-NC-ND 4.0.) 